\title{
Photoinduced phase-matched second-harmonic generation in azodye-doped polymer films
}

\section{$\operatorname{AUTHOR}(\mathrm{S}):$}

Si, Jinhai; Qiu, Jianrong; Kitaoka, Kenji; Hirao, Kazuyuki

\section{CITATION:}

Si, Jinhai ... [et al]. Photoinduced phase-matched second-harmonic generation in azodyedoped polymer films. JOURNAL OF APPLIED PHYSICS 2001, 89(4): 2029-2032

\section{ISSUE DATE:}

2001-02-15

URL:

http://hdl.handle.net/2433/39698

\section{RIGHT:}

Copyright 2001 American Institute of Physics. This article may be downloaded for personal use only. Any other use requires prior permission of the author and the American Institute of Physics. 


\title{
Photoinduced phase-matched second-harmonic generation in azodye-doped polymer films
}

\author{
Jinhai $\mathrm{Si}^{\mathrm{a})}$ and Jianrong Qiu \\ Photon Craft Project, ICORP, JST, Keihanna-plaza, Super-lab 2-5, 1-7 Hikaridai, Seika-cho, \\ Kyoto 619-0237, Japan \\ Kenji Kitaoka \\ Hirao Active Glass Project, ERATO, JST, Keihanna-plaza, Super-lab 2-6, 1-7 Hikaridai, Seika-cho, \\ Kyoto 619-0237, Japan \\ Kazuyuki Hirao \\ Division of Material Chemistry, Faculty of Engineering, Kyoto University, Sakyo-ku Kyoto 606-8501, Japan
}

(Received 31 July 2000; accepted for publication 3 December 2000)

\begin{abstract}
Phase-matched second-harmonic generation (SHG) in azodye-doped polymethylmethacrylate films was demonstrated using the nonresonant optical poling technique. During the seeding process, samples were irradiated simultaneously by coherent superposition of the fundamental and the second-harmonic light of a femtosecond laser. Optical poling experiments were performed at three wavelengths of 1500, 1320, and $1300 \mathrm{~nm}$, respectively. The quadratic dependence of SHG on the film thickness showed that a $\chi^{(2)}$ grating that satisfied the phase-matching condition for SHG was optically induced in the polymer films. The SHG conversion efficiency of a $105 \mu \mathrm{m}$ thick film was estimated to be about $2 \%$. A relaxation retardation effect of the photoinduced $\chi^{(2)}$ was also observed in the thick films. (C) 2001 American Institute of Physics. [DOI: 10.1063/1.1343890]
\end{abstract}

\section{INTRODUCTION}

Second-order nonlinear optical (NLO) polymers are very attractive for applications in optical communications and in high-density optical data storage. ${ }^{1,2}$ Poling techniques are usually used to realize the second-order NLO function of the polymer films. There are three kinds of poling techniques available at present, thermal-assisted electric-field poling, ${ }^{3}$ photoassisted electric-field poling, ${ }^{4}$ and all-optical poling. ${ }^{5}$ All-optical poling has been demonstrated in azoaromatic acrylic copolymers using a dual frequency laser. ${ }^{5-7}$ The physical mechanism of the effect consists of two processes: orientational hole burning and reorientation of azodye molecules. ${ }^{6,8}$ Under the excitation of a fundamental light along with its second-harmonic light, orientational hole burning of azodye molecules occurs through the interference of two-photon absorption at the fundamental frequency and one-photon absorption at the doubling frequency. Orientational hole burning is followed by reverse trans-cis-transisomerization, which finally leads to a net permanent polar orientation of molecules. This technique possesses some of the following advantages: phase matching for secondharmonic generation (SHG) can automatically be achieved, no electrodes are required, and micropattering of the secondorder susceptibility can be simply achieved by scanning the focal area over the sample surface.

There were, however, two problems to overcome for the application of this poling technique to photonic devices: one was the relaxation of the induced polar orientation of azodye molecules, and the other was low SHG conversion efficiency. In order to solve the former, we presented a thermal-

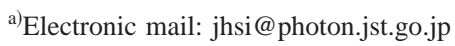

assisted optical poling, and successfully achieved thermosetting enhancement of the induced polar orientation stability in polymer films and glasses. ${ }^{9,10}$ In a resonant optical excitation process, it appears to be difficult for the SHG conversion efficiency to benefit from phase matching achieved automatically in large propagation distances due to the absorption of samples. Recently, we reported all-optical poling of azodyedoped polymethylmethacrylate (PMMA) films using the $1500 \mathrm{~nm}$ fundamental and $750 \mathrm{~nm}$ second-harmonic light of a femtosecond laser. ${ }^{11}$ Although the SHG conversion efficiency still was low due to the use of thin films only several micrometers thick, the nonresonant excitation should permit the use of larger film thickness, which should in turn result in a significant increase of the conversion efficiency. In this article, we experimentally demonstrate phase-matched SHG in thick films using the nonresonant optical-poling technique. Optical poling was performed at three different conditions in which wavelengths of two seed light beams were set, respectively, at 1500 and 750, 1320 and 660, and 1300 and $650 \mathrm{~nm}$. A quadratic dependence of SHG on the film thickness and a relaxation retardation effect of the photoinduced $\chi^{(2)}$ were observed in the films.

\section{EXPERIMENTS}

\section{A. Samples}

The samples used in this study were prepared by dissolving to a $15 \%$ mass ratio purified disperse red 1 (DR1) and medium molecular weight PMMA in telrahydorofuran. The films were spin deposited onto glass substrates, and dried for several hours at $160^{\circ} \mathrm{C}$. Films with different thicknesses were prepared by adjusting the viscosity of the solution and the revolutions per minute of the spin coater. The absorption 


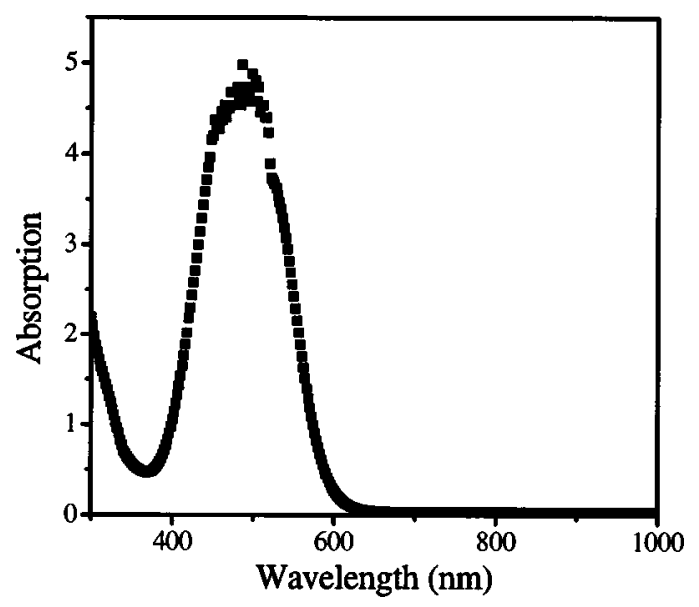

FIG. 1. Absorption spectrum of an azodye-doped PMMA film.

spectrum of a $1.3 \mu \mathrm{m}$ thick film is shown in Fig. 1, from which one can see that the absorption band peaks at $480 \mathrm{~nm}$, and the film is almost transparent at wavelengths larger than $650 \mathrm{~nm}$.

\section{B. Experimental setup}

A Ti:sapphire laser system with an optical parametric amplifier (OPA) which emitted 150 fs laser pulses at a repetition rate of $1 \mathrm{kHz}$ was used for all-optical poling of the films, and the wavelength of the light from the OPA could be tuned from 300 to $3000 \mathrm{~nm}$. The average power of the laser was about $50 \mathrm{~mW}$ at wavelengths ranging from 1300 to 1500 $\mathrm{nm}$. A seed beam $\omega$ for all-optical poling was split from the source beam and passed through a time-delay device and a $\lambda / 2$ plate to control the path length and the polarization of the beam, respectively. Another beam separate from the source beam was frequency doubled by a KDP crystal, which served as another seed beam, and is denoted seed beam $2 \omega$ here. The two collinear seed beams were introduced into the film sample through a $6 \mathrm{~cm}$ focal-length quartz lens. We achieved time superposition of pulses between the two seed beams by adjusting the time-delay device and observing the optical Kerr effect of $\mathrm{CS}_{2}$. During the optical-poling process, the two seed beams were introduced simultaneously into the film sample; during the probe, beam $2 \omega$ was blocked by a shutter and only beam $\omega$ remained incident. The SHG signals of beam $\omega$ were detected by a photomultiplier and observed and averaged by an oscilloscope. Beam $\omega$ passing through the sample was blocked by a heat-absorbing filter placed behind the sample, and allowed only the SHG signal to pass through it. Typical fluences of the two seed beams at the sample were approximately $1.5 \mathrm{~mJ} / \mathrm{cm}^{2}$ for beam $\omega$ and $0.03 \mathrm{~mJ} / \mathrm{cm}^{2}$ for beam $2 \omega$, respectively.

\section{RESULTS AND DISCUSSION}

\section{A. Dependence of SHG on film thickness}

By adjusting the delay device, we measured the dependence of photoinduced SHG on the temporal correlation of the two seed beams, and observed that all-optical poling of the films could been performed only when the two seed beams were coherently superpositioned. This indicated that all-optical poling resulted from coherent excitation of the two seed lights for azodye molecules in the films. To confirm that the SHG signals were from phase-matched SHG, we measured the dependence of the SHG signal intensity versus the sample thickness. Figure 2(a) shows the results measured for the films written by two seed beams with wavelengths of 1500 and $750 \mathrm{~nm}$, where all of the measurements were made after the photoinduced $\chi^{(2)}$ of the films increased to their saturation values. It can be seen from Fig. 2 that the SHG signals increase proportionally to the square of the film thickness. It is well known that for a thick film of thickness of larger than SHG coherent length (the coherent length of these films is about $12.5 \mu \mathrm{m}$ ), the quadratic thickness dependence of the SHG intensity occurs only in the phase-matched condition. A peak conversion efficiency of $2 \%$ was observed for the $105 \mu \mathrm{m}$ film and a fundamental light intensity of 9 $\mathrm{GW} / \mathrm{cm}^{2}$ incident on the film. Obviously, this high conversion efficiency benefited from phase-matched SHG in the thick film. We also observed that the SHG intensity of the films departed from the quadratic length dependence when the thickness of the films was larger than $105 \mu \mathrm{m}$. This probably is due to the group velocity mismatch between seed beam $\omega$ and seed beam $2 \omega$. The group velocity mismatch causes the $\omega$ seed pulse to displace against the $2 \omega$ seed pulse as they propagate along in the film. This reduces the effective interaction length and decreases the optical-poling efficiency.

Moreover, results similar to those described above were also observed when the wavelengths of the two seed beams were set, respectively, at 1300 and 650 and 1320 and 660 $\mathrm{nm}$. The experimental results for both cases are shown in Figs. 2(b) and 2(c), respectively.

\section{B. Dependence of the relaxation of the photoinduced $\chi^{(2)}$ on film thickness}

As reported by us in a previous study, ${ }^{11}$ photoinduced $\chi^{(2)}$ of DR1-doped PMMA films had obvious relaxation. We found here, however, that the relaxation rate decreased with increasing thickness of the films, and when the SHG signal intensity from the thick films reached a certain level, the relaxation of the photoinduced $\chi^{(2)}$ was completely suppressed. Figure 3 shows the typical relaxation measured for the three films with thicknesses of $105,40.5$, and $8.2 \mu \mathrm{m}$. The films were written by the 1500 and $750 \mathrm{~nm}$ seed beams. All of the measurements were made after the photoinduced $\chi^{(2)}$ of the films increased to their saturation values. It can be found from Fig. 3 that for the $105 \mu \mathrm{m}$ film no measurable change in the SHG signals occurred over a period of $30 \mathrm{~min}$. And the SHG signal intensity of the film was also observed to be comparable with the $2 \omega$ seed intensity.

The relaxation retardation effect of the photoinduced $\chi^{(2)}$ for the $105 \mu \mathrm{m}$ film is probably related to the seeding for the film by the $\omega$ readout light and the SHG signal light (we call it "self-seeding" here). In case of the $105 \mu \mathrm{m}$ film, this self-seeding could counteract the relaxation effect during the readout process because the SHG signal light of the film was strong enough to completely suppress the relaxation of 

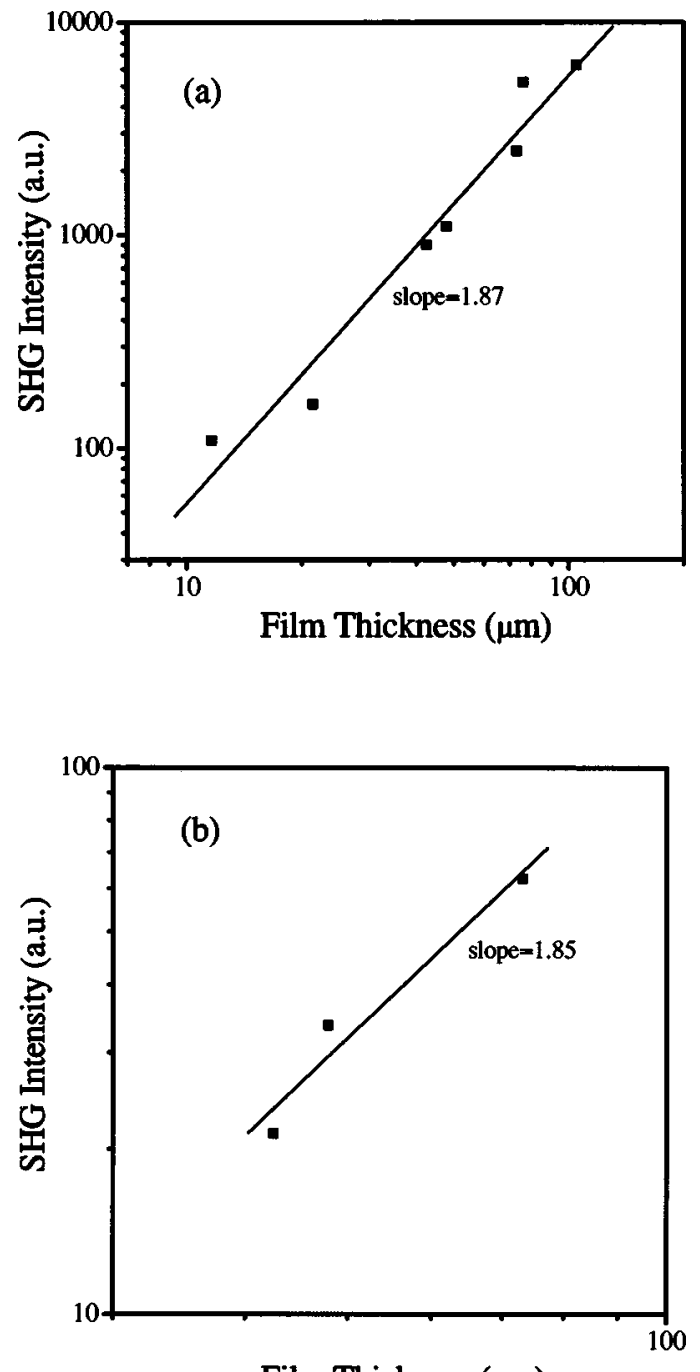

Film Thickness ( $\mu \mathrm{m})$

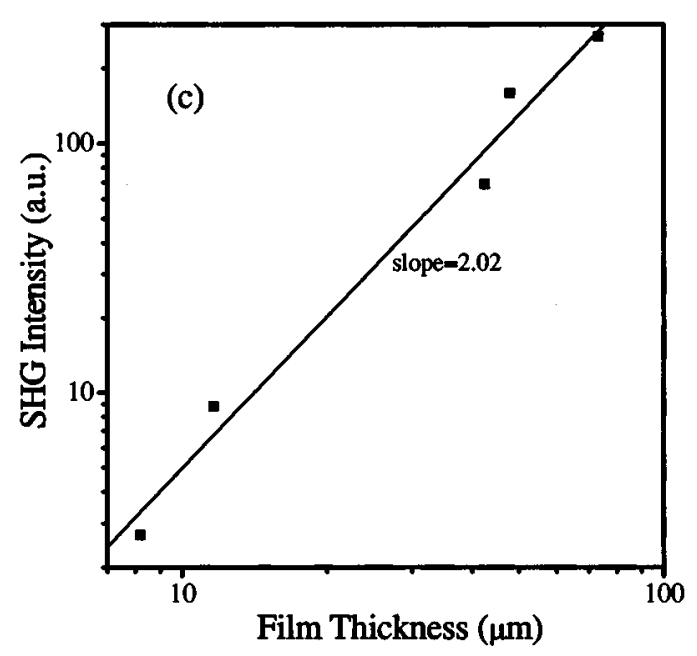

FIG. 2. Log- $\log$ plots with a linear fit of a slope near 2, displaying SHG intensity dependence on the film thickness squared. In the optical-poling processes wavelengths of the $\omega$ and $2 \omega$ seed beams were set at, respectively, 1500 and 750 (a), 1320 and 660 (b), and 1300 and $650 \mathrm{~nm}$ (c).

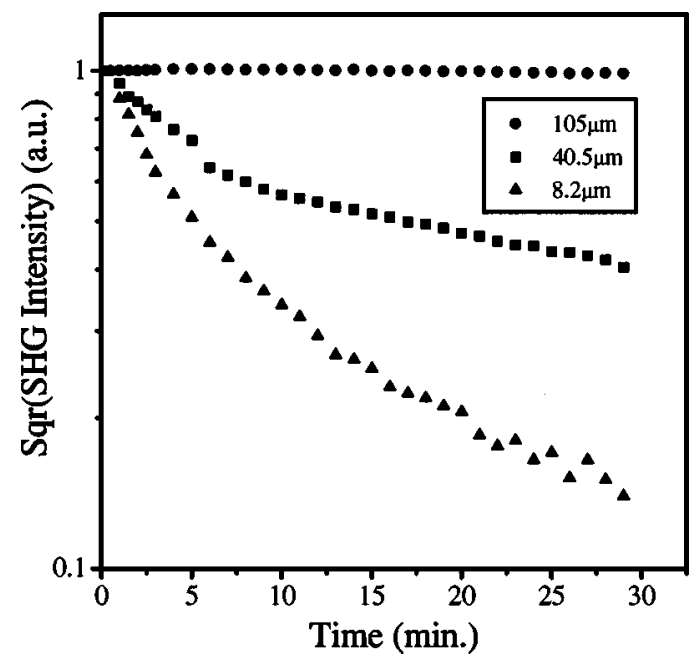

FIG. 3. Relaxations of the photoinduced $\chi^{(2)}$ of three films with different thicknesses. The films were written optically by two seed beams with wavelengths of 1500 and $750 \mathrm{~nm}$.

the photoinduced $\chi^{(2)}$. In contrast, for the two films with thicknesses of 40.5 and $8.2 \mu \mathrm{m}$, the SHG signal intensities of the films were too low to completely suppress the relaxation.

\section{Tensor analysis of the photoinduced $\chi^{(2)}$}

For tensor analysis, we performed experiments to measure the $x$ and the $y$ components of the SHG signals by keeping the back polarizer fixed in the vertical $(x)$ or horizontal (y) position and rotating the readout-beam polarization. Figure 4 displays the tensor curve for the $x$ component for a 73 $\mu \mathrm{m}$ film written by the 1300 and $650 \mathrm{~nm}$ seed beams. The tensor curve was fitted by general polarization expressions as the following: ${ }^{12}$

$$
\begin{aligned}
& \left|E_{x}^{2 \omega}\right|^{2} \propto\left|\chi_{x x x}^{(2)} \cos ^{2} \theta+2 \chi_{x x y}^{(2)} \cos \theta \sin \theta+\chi_{x y y}^{(2)} \sin ^{2} \theta\right|^{2}, \\
& \left|E_{y}^{2 \omega}\right|^{2} \propto\left|\chi_{y y y}^{(2)} \sin ^{2} \theta+2 \chi_{y y x}^{(2)} \cos \theta \sin \theta+\chi_{y x x}^{(2)} \cos ^{2} \theta\right|^{2},
\end{aligned}
$$

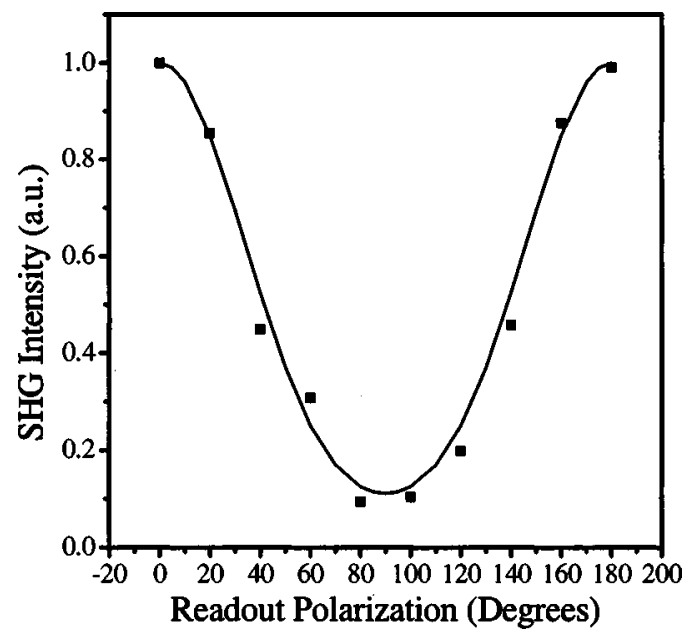

FIG. 4. Tensor data and fits for the $x$ component of SHG signals from the photoinduced $\chi^{(2)}$ of the $73 \mu \mathrm{m}$ film. The films were written optically by two seed beams with wavelengths of 1300 and $650 \mathrm{~nm}$. 
where $\theta$ is the readout beam polarization measured with respect to the vertical $x$ axis. The best fits gave

$$
\chi_{x x x}^{(2)} / \chi_{x y y}^{(2)} \approx 3
$$

and

$$
\chi_{x x y}^{(2)}=0
$$

From the measurements for the $y$ component, we obtained

$$
\chi_{x x x}^{(2)} / \chi_{y y y}^{(2)}>12
$$

and

$$
\chi_{y y x}^{(2)}=0 .
$$

The results of this tensor analysis indicate that for collinearly polarized seed beams the photoinduced $\chi^{(2)}$ in the film exhibited axial symmetry along the seed beam polarization direction.

Here, we could not measure the magnitudes of the photoinduced $\chi^{(2)}$ of the films with femtosecond pulses because of the limit of our experimental conditions. To show the capability of optical poling for the films using a nonresonant femtosecond laser, we measured the SHG intensity ratio for the $73 \mu \mathrm{m}$ film, $I_{s} / I_{q}$, where $I_{s}$ and $I_{q}$ are, respectively, the SHG intensities of the film and a Y-cut quartz for the same readout wavelength and readout power. During the seeding processes, wavelengths of two seed beams were set at, respectively, 1300 and 650, 1320 and 660, and 1500 and $750 \mathrm{~nm}$. The square root of the SHG intensity ratio between the film and the quartz, $\left(I_{s} / I_{q}\right)^{1 / 2}$, was measured to be 6.6, $7.5,18.6$, respectively, for fundamental wavelengths of 1300 , 1320 , and $1500 \mathrm{~nm}$. These values are near to that of optical poling made using a resonant nanosecond laser, ${ }^{9}$ indicating effective optical poling also can be achieved even in non- resonant excitation when a femtosecond laser with high peak power is used.

\section{CONCLUSION}

We experimentally demonstrated phase-matched SHG in azodye-doped PMMA films using the nonresonant opticalpoling technique. Experiments were performed at three wavelengths, 1500, 1320, and $1300 \mathrm{~nm}$. Square dependence of SHG on the film thickness was observed, and the SHG conversion efficiency of the $105 \mu \mathrm{m}$ film was estimated to be about $2 \%$. The relaxation retardation effect of the photoinduced $\chi^{(2)}$ also was observed as the film thickness was increased. It is expected that this study will promote the application of all-optical poling in photonic devices.

\footnotetext{
${ }^{1}$ Molecular Nonlinear Optics-Material, Physics and Devices, edited by J. Zyss (Academic, San Diego, CA, 1994).

${ }^{2}$ Nonlinear Optical Properties of Polymers, edited by A. J. Heeger, J. Orenstein, and D. R. Ulrich (Materials Research Society, Pittsburgh, PA, 1988).

${ }^{3}$ K. D. Singer, J. E. Sohn, and S. J. Lalama, Appl. Phys. Lett. 49, 248 (1986).

${ }^{4}$ Z. Sekkat and M. Dumont, Appl. Phys. B: Photophys. Laser Chem. B45, 486 (1992).

${ }^{5}$ F. Charra, F. Kajzar, J.-M. Nunzi, P. Raimond, and E. Idiart, Opt. Lett. 18, 941 (1993).

${ }^{6}$ W. Chalupczak, C. Fiorini, F. Charra, J.-M. Nunzi, and P. Raimond, Opt. Commun. 126, 103 (1996).

${ }^{7}$ C. Fiorini, F. Charra, J.-M. Nunzi, and P. Raimond, J. Opt. Soc. Am. B 14, 1984 (1997).

${ }^{8}$ F. Charra, F. Devaux, J.-M. Nunzi, and P. Raimond, Phys. Rev. Lett. 68, 2440 (1992).

${ }^{9}$ J. Si, K. Kitaoka, T. Mitsuyu, P. Ye, and K. Hirao, J. Appl. Phys. 85, 8018 (1999).

${ }^{10}$ K. Kitaoka, J. Si, T. Mitsuyu, and K. Hirao, J. Ceram. Soc. Jpn. 107, 522 (1999).

${ }^{11}$ K. Kitaoka, J. Si, T. Mitsuyu, and K. Hirao, Appl. Phys. Lett. 75, 157 (1999).

${ }^{12}$ T. J. Driscoll and N. M. Lawandy, J. Opt. Soc. Am. B 11, 355 (1994).
} 